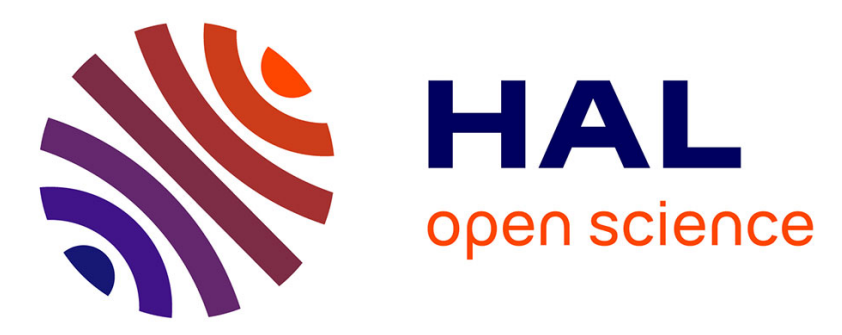

\title{
Analysis and Design of Bessel Beam Launchers: Transverse Polarization
}

S.C. Pavone, Mauro Ettorre, Massimiliano Casaletti, M. Albani

\section{To cite this version:}

S.C. Pavone, Mauro Ettorre, Massimiliano Casaletti, M. Albani. Analysis and Design of Bessel Beam Launchers: Transverse Polarization. IEEE Transactions on Antennas and Propagation, 2021, 69 (8), pp.5175-5180. 10.1109/TAP.2021.3060049 . hal-03195892

\section{HAL Id: hal-03195892 https://hal.science/hal-03195892}

Submitted on 16 Apr 2021

HAL is a multi-disciplinary open access archive for the deposit and dissemination of scientific research documents, whether they are published or not. The documents may come from teaching and research institutions in France or abroad, or from public or private research centers.
L'archive ouverte pluridisciplinaire HAL, est destinée au dépôt et à la diffusion de documents scientifiques de niveau recherche, publiés ou non, émanant des établissements d'enseignement et de recherche français ou étrangers, des laboratoires publics ou privés. 


\title{
Communication Analysis and Design of Bessel Beam Launchers: Transverse Polarization
}

\author{
Santi C. Pavone, Member, IEEE, Mauro Ettorre, Senior Member, IEEE, \\ Massimiliano Casaletti, Member, IEEE, and Matteo Albani, Fellow, IEEE
}

\begin{abstract}
In this paper, we present a theoretical analysis, one design and fabrication of a limited-diffractive planar Bessel beam launcher, that exhibits a zeroth order Bessel profile in the transverse electric field component with respect to the $z$ propagation axis. The launcher is designed by synthesizing a finite zeroth order, first kind Hankel aperture distribution, polarized along a fixed polarization unit vector. The field radiated by such an aperture distribution is derived by following an approximate model based on geometric theory of diffraction, thus allowing to highlight the relevant wave constituents involved in transverse Bessel beam generation, also including the effect of aperture truncation on the radiated beam. Moreover, the theoretical analysis has been profitably applied to the design of a circularpolarized planar transverse Bessel beam launcher by means of a slotted radial waveguide. A prototype of right-handed circular polarized (RHCP) transverse Bessel beam launcher has been then fabricated at $f=30 \mathrm{GHz}$. The measured transverse electric field component shows a satisfactory agreement with full-wave numerical simulations.
\end{abstract}

Index Terms-Bessel beams, non-diffractive beams, RLSA, circular polarization, near-field focusing.

\section{INTRODUCTION}

In the last decade, non-diffractive beams, i.e. ideal solutions of scalar Helmholtz equation in cylindrical coordinates that are theoretically not affected by the physical phenomenon of diffraction, have gained increasing attention among engineers and physicists, due to their remarkable features that can profitably find applications at microwaves and millimeter waves. Specifically, the dramatic reduction of beam diffraction along the propagation direction results in well-collimated beams, that can be applied in medical imaging [1]-[5], in ground penetrating radars (GPR) [6], [7], in small particle manipulation, just to mention the most relevant of them.

Among non-diffractive solutions of Helmholtz equation, Bessel beams are of paramount importance both from historical and engineering viewpoints. Introduced by Durnin in 1987 [8], [9], at the beginning they have been mainly studied in optics [10]-[14], since so-called axicon lenses were available

Santi C. Pavone is with the Department of Electrical, Electronics and Computer Engineering (DIEEI), University of Catania, Viale Andrea Doria 6, 95125 Catania, Italy. (e-mail: santi.pavone@unict.it).

Mauro Ettorre is with the Institut d'Electronique et des Technologies du numéRique (IETR), UMR CNRS 6164, Université de Rennes 1, 35000 Rennes, France.

Massimiliano Casaletti is with the Sorbonne Université, Laboratoire d'Électronique et Électromagnétisme (L2E), F-75005, Paris, France.

Matteo Albani is with the Department of Information Engineering and Mathematics (DIISM), University of Siena, Via Roma 56, 53100 Siena, Italy.

Santi C. Pavone wishes to thank the PON-AIM "Attraction and International Mobility" project, granted by the Italian Ministry of Education and Scientific Research (MUR).

Manuscript received XX XX, 2020; revised XX XX, 2020. to generate Bessel beams, i.e. stationary waves in cylindrical coordinates, as the superposition of both inward and outward cylindrical traveling waves. However, the generation of Bessel beams at lower frequencies, such as microwaves/millimeter waves, has been considered cumbersome up to more recent years [15]-[21], mainly due to the fact that Bessel beam generation by planar devices at those frequencies in general requires a somewhat involved electromagnetic (EM) analytical formulation, but above all because planar and lightweight solutions able to radiate Bessel beams were not easily available, due to technological issues. Indeed, mainly in the last

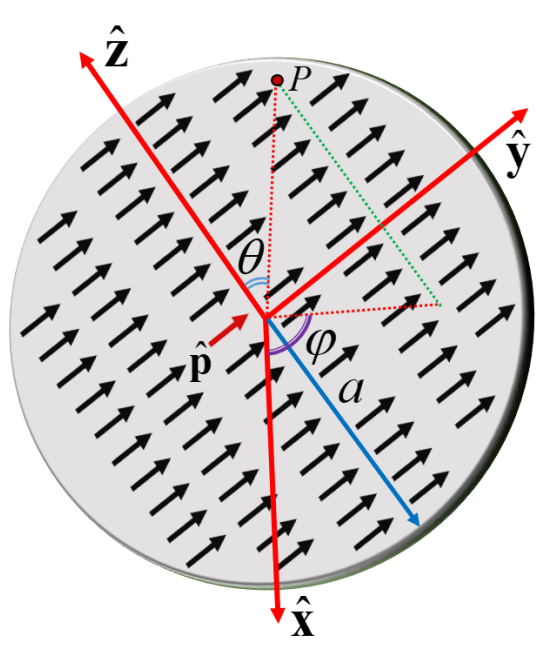

Fig. 1. Finite equivalent magnetic current distribution of radius $a$, shaped as a zeroth order first kind Hankel function, and polarized along an arbitrary fixed polarization unit vector $\hat{\mathbf{p}}$.

decade different advanced approaches (i.e., holography [22], [23], leaky-wave theory applied to focusing [23], [24], etc.) have been profitably adopted for planar Bessel beam launcher design. It is well-established [8], [20] that an exact nondiffractive beam cannot exist, since it should carry infinite energy (i.e., like plane waves), that in turn imply infinite radiating apertures. However, finite radiating apertures can radiate a Bessel beam in a limited spatial region, up to a certain distance called non-diffractive range, beyond which beam starts spreading out and loses its collimation property. Hence, Bessel beam launchers are used only for near-field applications, thus complicating also the analytical formulation for describing them.

In this paper, we present the analysis and characterization of a Bessel beam launcher that implements a zeroth order 
Bessel function aperture distribution in the transverse electric field component with respect to the propagation $z$-axis, by considering a magnetic current distribution oriented along a fixed unit vector $\hat{\mathbf{p}}$ (in the following, for the sake of simplicity, we will simply refer also to transverse Bessel beam launchers). The proposed approach appears more convenient if compared to that discussed in [20] for different reasons. Indeed, by removing the restriction of azimuthally-oriented equivalent magnetic current distribution, a larger number of geometrical degrees of freedom in aperture field distribution optimization are available, together with the additional possibility to synthesize circular-polarized (CP) fields. Moreover, since EM field transverse component spatially decays slowly from the aperture with respect to the longitudinal one, the achievable non-diffractive range and spatial collimation of the resulting $\mathrm{CP}$ Bessel beam launcher are in general improved, due in turn to the fact that smaller axicon angles $\left(\theta_{a} \approx 15^{\circ}\right)$ can be achieved with respect to [20]. Although in [19] a higher nondiffractive range $(\approx 85 \lambda)$ has been achieved in simulation, the proposed design was not so easy to be fabricated, especially for the adopted substrate (foam, $\epsilon_{r}=1.04$ ), not suitable for standard PCB process. The optimization in presence of dielectrics of higher permittivity is sometimes cumbersome, hence the achieved results can be considered satisfactory, leading to fabrication easiness.

To highlight relevant wave constituents involved in description of transverse Bessel beam radiation, an approximate EM approach based on geometric theory of diffraction (GTD) is presented in the following, by taking into account also the effect of radiating aperture truncation. The EM field radiated by a zeroth order first kind aperture distribution oriented along a fixed unit vector $\hat{\mathbf{p}}$ is split in two ray-optical contributions, namely the geometrical optics (GO) and the diffracted (D) ones. Such an approach allows to precisely define spatial regions in which a transverse Bessel beam can exists, and to highlight also space-wave contributions and edge-diffracted rays arising from the aperture rim. It thus provides physical insight on transverse Bessel beam generation. The approach in the present work completes the analysis of Bessel beam radiation, outlined in [25], by an inward cylindrical traveling wave aperture distribution polarized along a fixed unit vector. There, a rigorous analytical approach has been applied only in the case of an infinite radiating aperture. Instead, a detailed description of truncated aperture radiation in terms of edge-diffracted waves is not present. Moreover, in [25] no prototypes of transverse Bessel beam launcher are presented. On the other hand, this paper reveals more physical insights on transverse Bessel beam generation than [26], in which wideband performances of Bessel beam launchers are discussed for localized pulse efficient radiation, without any detailed GTD description.

The paper is organized as follows. In Section II, a GTDbased EM analytical model is discussed, to describe the relevant physical wave constituents involved in transverse Bessel beam generation by a zeroth order, first kind Hankel aperture distribution, polarized along an arbitrary transverse unit vector $\hat{\mathbf{p}}$. Then, in Section III the optimization algorithm developed for the synthesis of such an aperture distribution by means a radial parallel-plate waveguide (PPW), loaded by a huge number of slot pairs, is presented. Finally, in Section IV details about prototype fabrication and measurements in the bandwidth $29-31 \mathrm{GHz}$ are shown. In the end, conclusions are drawn.

\section{FORMULATION FOR FINITE TRANSVERSE-POLARIZED BESSEL BEAM LAUNCHERS}

The geometry of the EM problem is shown in Fig. 1, in which a finite circular equivalent magnetic current distribution oriented along a fixed polarization unit vector $\hat{\mathbf{p}}$ is schematically shown. A Cartesian coordinate system is defined with origin at the aperture center, $x$ and $y$ axes on the aperture, and $z$ axis orthogonal to the aperture, pointing the boresight direction. Beside Cartesian coordinates, an observation point

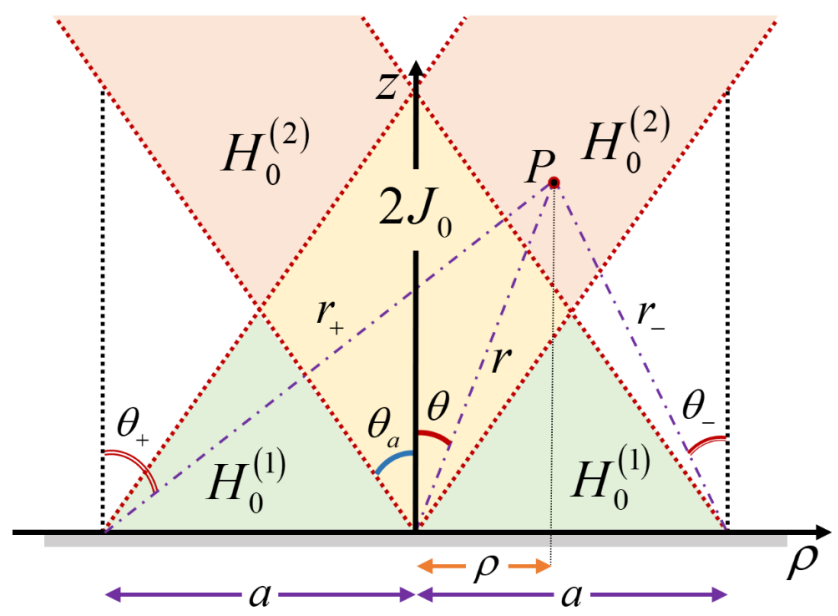

Fig. 2. Reference system for GTD analysis of Bessel beam generation by a finite inward cylindrical traveling wave magnetic current distribution, polarized along a fixed unit vector. The colored regions are associated to the transverse electric field component. The transverse Bessel beam (i.e., shaped as a zeroth order Bessel function) is radiated in the yellow rhomboidal section conical region around the aperture axis of symmetry. GO shadow boundaries are denoted by red dotted lines.

$P$, denoted by the vector $\mathbf{r}$, is also expressed by its standard cylindrical and spherical coordinates. Moreover, in Fig. 2 two auxiliary reference systems, centered at the aperture edges, are introduced, which are suitable to express edge-diffracted waves. The usual $e^{j \omega t}$ time-harmonic convention is assumed and suppressed throughout the paper.

In [20], an azimuthally-polarized (i.e., $\phi$-oriented) finite magnetic current distribution has been imposed over the aperture and shaped as a first order, first kind Hankel function. Such a distribution radiates a Bessel beam (i.e., shaped as a zeroth order Bessel function) in the longitudinal (i.e., $z$ ) electric field component. Instead, here we assume a magnetic current distribution on the aperture of the form of a zeroth order, first kind Hankel distribution, polarized along the $\hat{\mathbf{p}}$ unit vector, namely

$$
\mathbf{M}(\rho)=H_{0}^{(1)}\left(k_{\rho a} \rho\right) \hat{\mathbf{p}},
$$

in which $k_{\rho a}=k \sin \theta_{a}$ is the imposed radial wavenumber on the radiating aperture, $k$ the free-space wavenumber, and 
$\theta_{a}$ the so-called axicon angle [20]. In the following, it will be rigorously proven that such an aperture distribution can radiate a Bessel beam in the transverse electric field component.

The field radiated by such an aperture distribution can be calculated by using the theory of vector potentials [27]. Since in the present case only a magnetic current distribution is considered over the aperture, only the vector potential $\mathbf{F}(\mathbf{r})=F(\mathbf{r}) \hat{\mathbf{p}}$ is required for electric field calculation, that is

$$
\mathbf{E}(\mathbf{r})=-\frac{1}{\epsilon} \nabla \times \mathbf{F}(\mathbf{r})=-\frac{1}{\epsilon} \nabla F(\mathbf{r}) \times \hat{\mathbf{p}} .
$$

Due to the azimuthal symmetry of the magnetic current distribution in (1), the problem of field calculation can be simplified in the spectral domain. Hence, the potential $F$ can be recast in the spectral $k_{\rho}$-plane, being $k_{\rho}$ the radial wavenumber (refer to the Appendix A for details), as

$$
F(\mathbf{r})=\frac{\epsilon}{4 \pi j} \int_{\infty e^{-j \pi}}^{+\infty} \tilde{M}\left(k_{\rho}\right) H_{0}^{(2)}\left(k_{\rho} \rho\right) \frac{e^{-j k_{z} z}}{k_{z}} k_{\rho} d k_{\rho},
$$

where the integration contour extends along the lower shore of the branch-cut on the negative real axis due to the zeroth order, second kind Hankel function (see [20], Fig. 2), and $k_{z}=\sqrt{k^{2}-k_{\rho}^{2}}$ the longitudinal wavenumber $\left(\Im\left\{k_{z}\right\}<0\right.$ on the top Riemann sheet). Moreover, $\tilde{M}\left(k_{\rho}\right)$ is the Hankel transform of the finite magnetic current distribution imposed over the aperture, namely

$$
\tilde{M}\left(k_{\rho}\right)=2 \pi \int_{0}^{a} M(\rho) J_{0}\left(k_{\rho} \rho\right) \rho d \rho .
$$

in which $a$ is the aperture radius (1). By using (1) in (4), $\tilde{M}\left(k_{\rho}\right)$ can be evaluated in closed form. In particular, as outlined in [20], the asymptotic evaluation of the vector potential (3), relevant to the assumed aperture distribution, permits to highlight the physics of transverse Bessel beam generation by means of a simple ray-optical interpretation. To this end, we consider the magnetic current as the sum of three contributions,

$$
\tilde{M}\left(k_{\rho}\right)=\tilde{M}_{\infty}\left(k_{\rho}\right)+\tilde{M}_{+}\left(k_{\rho}\right)+\tilde{M}_{-}\left(k_{\rho}\right),
$$

being $\tilde{M}_{\infty}\left(k_{\rho}\right)$ the spectrum of an infinite (i.e., extending over a full infinite plane) magnetic current distribution, that is

$$
\tilde{M}_{\infty}\left(k_{\rho}\right)=-\frac{4 j}{k_{\rho}^{2}-k_{\rho a}^{2}},
$$

whereas

$$
\begin{aligned}
\tilde{M}_{ \pm}\left(k_{\rho}\right)= & \frac{\pi a}{k_{\rho}^{2}-k_{\rho a}^{2}}\left[k_{\rho a} H_{1}^{(1)}\left(k_{\rho a} a\right) H_{0}^{(2,1)}\left(k_{\rho} a\right)\right. \\
& \left.-k_{\rho} H_{0}^{(1)}\left(k_{\rho a} a\right) H_{1}^{(2,1)}\left(k_{\rho} a\right)\right],
\end{aligned}
$$

are additional spectral terms that account for aperture finiteness. Indeed, for $a \rightarrow+\infty$, such terms vanish, whereas the contribution (6) does not depend on the aperture size.

The integral in (3) can be approximated by using the steepest descent path (SDP) method, as outlined in [16]. The contribution due to an infinite radiating aperture can be split in its GO and D contributions, namely

$$
F_{\infty}(\mathbf{r})=F_{\infty}^{G O}(\mathbf{r})+F_{\infty}^{D}(\mathbf{r}),
$$

in which the GO term is calculated as the sum of the residues of (3), when $\tilde{M}\left(k_{\rho}\right)=\tilde{M}_{\infty}\left(k_{\rho}\right)$ (i.e., infinite aperture contribution), at the poles $k_{\rho}= \pm k_{\rho a}$, so that

$$
\begin{aligned}
& F_{\infty}^{G O}(\mathbf{r})= \epsilon \frac{e^{-j k_{z a} z}}{j k_{z a}} \\
& \cdot\left[H_{0}^{(2)}\left(k_{\rho a} \rho\right) U\left(\theta_{a}-\theta\right)+H_{0}^{(1)}\left(k_{\rho a} \rho\right)\right],
\end{aligned}
$$

in which $k_{z a}=k \cos \theta_{a}$. It is worth noting that the pole at $k_{\rho}=k_{\rho a}$ is encountered by the integration path deformation onto the SDP only when the saddle point $k_{\rho}^{s}=k \sin \theta$ is smaller than $k \sin \theta_{a}$ [20] (see Fig. 2), hence the unitary step function $U\left(\theta_{a}-\theta\right)$ has been introduced, which vanishes for negative argument. The GO contribution (9) of an infinite aperture comprises two conical waves, both propagating in the $z$-direction away from the aperture, one propagating outwardly and one inwardly in the radial direction. The former is bounded in a conical region $\theta<\theta_{a}$, whose tip is at the aperture center; in such a region, the interference of such two wave constituents creates a cylindrical standing wave, i.e., the Bessel beam.

As concerns the SDP contribution to the vector potential $F_{\infty}^{D}(\mathbf{r})$, (3) has to be arranged in such a way to isolate a slowly-varying integrand and a rapidly-oscillating phase term. By multiplying and dividing the integrand in (3) by the conjugate asymptotic $\left(k_{\rho} \rho \gg 1\right)$ phase behaviour of the function $H_{0}^{(2)}\left(k_{\rho} \rho\right)$, it can be split as the product of a slowly-varying function, namely $\tilde{M}_{\infty}\left(k_{\rho}\right) H_{0}^{(2)}\left(k_{\rho} \rho\right) e^{j k_{\rho} \rho}$, and a rapidly-oscillating phase term, $e^{-j\left(k_{\rho} \rho+k_{z} z\right)}$, according to the theory of asymptotic evaluation of integrals [28]. Hence, the SDP contribution due to the infinite aperture becomes

$$
F_{\infty}^{D}(\mathbf{r}) \sim \frac{2 \epsilon}{j \pi k^{2}\left(\sin ^{2} \theta-\sin ^{2} \theta_{a}\right)} \frac{e^{-j k r}}{r} .
$$

The latter asymptotic expression describes a spherical wave contribution (space wave) that decays as $1 / r$ from the aperture center, with an elevation pattern exhibiting a caustic at the GO shadow boundary cone $\theta= \pm \theta_{a}$, where the GTD fails.

The effect to the radiated field due to the aperture finiteness can be taken into account, in the GTD framework, by considering the edge-diffracted rays from the aperture circular rim. To this end, we consider the asymptotic evaluation of (3) when $\tilde{M}\left(k_{\rho}\right)=\tilde{M}_{ \pm}\left(k_{\rho}\right)$. Again, the asymptotic phase progression $e^{-j\left[k_{\rho}(\rho \mp a)+k_{z} z\right]}$ reveals a saddle point at $k_{\rho}^{s}=k \sin \theta_{ \pm}$, being $\tan \theta_{ \pm}=(\rho \pm a) / z$ and $r_{ \pm}=\sqrt{(\rho \pm a)^{2}+z^{2}}$ (Fig. 2), and the integral is calculated by deforming the integration path onto the SDP through the saddle point, as the sum of a residue contribution at the pole $k_{\rho a}=k \sin \theta_{a}$ and the integral on the SDP, which is asymptotically dominated by the saddle point contribution. Namely,

$$
F_{ \pm}(\mathbf{r})=F_{ \pm}^{G O}(\mathbf{r})+F_{ \pm}^{D}(\mathbf{r}),
$$

where also in this case the GO and $\mathrm{D}$ terms can be determined as

$$
F_{ \pm}^{G O}(\mathbf{r})=-\epsilon \frac{e^{-j k_{z a} z}}{j k_{z a}} H_{0}^{(2,1)}\left(k_{\rho a} \rho\right) U\left(\theta_{a} \mp \theta_{ \pm}\right),
$$


and

$$
F_{ \pm}^{D}(\mathbf{r}) \sim \mp \epsilon \sqrt{\frac{j}{2 \pi k^{3}}} \frac{H_{0}^{(1)}\left(k_{\rho a} a\right)}{\sin \theta_{ \pm} \mp \sin \theta_{a}} \sqrt{\frac{a}{\rho r_{ \pm}}} e^{-j k r_{ \pm}} .
$$

The former GO expressions (12) exhibit the same wave constituents (conical waves) appearing in the infinite aperture case (9), and cancel them out outside a shadow boundary cone $\theta_{ \pm}=\theta_{a}$. The latter asymptotic expressions describe edgediffracted waves arising at the aperture rim, whose spreading reveals an astigmatic wavefront with radii of curvature $r_{ \pm}$, i.e., the distance from the observation point to the diffraction point on the edge, and $\rho$, accordingly to GTD edge diffraction. The GTD expressions (13) exhibit an elevation pattern which is singular at $\theta_{ \pm}= \pm \theta_{a}$, that is the GO shadow boundary where they fail. In addition (13) are also singular at $\rho=0$, i.e., when observing on the $\mathrm{z}$-axis, which is a caustic of the edge-diffracted waves.

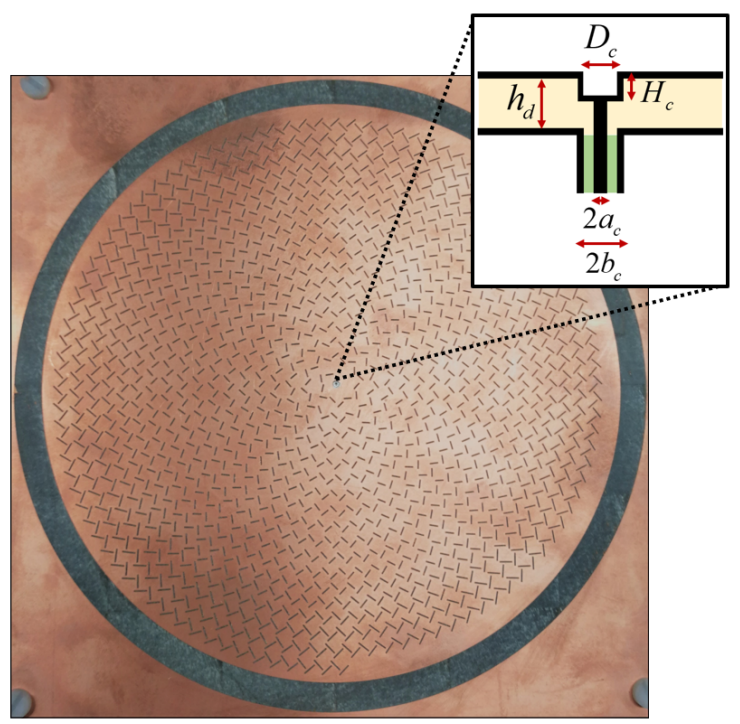

Fig. 3. Prototype of Bessel beam launcher at $f=30 \mathrm{GHz}$, synthesizing a RHCP zeroth order, first kind Hankel aperture distribution. In the inset, details of the transition used for impedance matching at input coaxial port.

All GO contributions to the vector potential can be then collected together to obtain

$$
F^{G O}(\mathbf{r})=F_{\infty}^{G O}(\mathbf{r})+F_{+}^{G O}(\mathbf{r})+F_{-}^{G O}(\mathbf{r}),
$$

from which the radiated GO electric field can be finally calculated by using (2) as

$$
\begin{aligned}
& \mathbf{E}^{G O}(\mathbf{r})= \\
& {\left[H_{0}^{(2)}\left(k_{\rho a} \rho\right) \hat{\mathbf{z}} \times \hat{\mathbf{p}}+\hat{\mathbf{p}} \cdot \hat{\boldsymbol{\phi}} \frac{k_{\rho a}}{j k_{z a}} H_{1}^{(2)}\left(k_{\rho a} \rho\right) \hat{\mathbf{z}}\right] .} \\
& e^{-j k_{z a} z}\left(U\left(\theta_{a}-\theta\right)-U\left(\theta_{a}-\theta_{+}\right)\right)+ \\
& {\left[H_{0}^{(1)}\left(k_{\rho a} \rho\right) \hat{\mathbf{z}} \times \hat{\mathbf{p}}+\hat{\mathbf{p}} \cdot \hat{\boldsymbol{\phi}} \frac{k_{\rho a}}{j k_{z a}} H_{1}^{(1)}\left(k_{\rho a} \rho\right) \hat{\mathbf{z}}\right] .} \\
& e^{-j k_{z a} z} U\left(-\theta_{-}-\theta_{a}\right),
\end{aligned}
$$

in which $\hat{\boldsymbol{\phi}}=-\sin \phi \hat{\mathbf{x}}+\cos \phi \hat{\mathbf{y}}$. The GO field radiated by the finite aperture comprises the same two conical waves constituents of the infinite aperture, but differently bounded by the aperture truncation. Namely, the radial inward conical wave exists in the conical region $\theta_{-}<\theta_{a}$ (green region in Fig. 2); the radial outward conical wave exists in the region $\theta_{+}>\theta_{a} \cap \theta<\theta_{a}$ (orange region in Fig. 2). In the overlapping of the two existence regions (yellow region in Fig. 2) the interference of the two conical waves creates the vector Bessel beam [29], namely

$$
\begin{aligned}
\mathbf{E}^{G O}(\mathbf{r}) & =\left[2 J_{0}\left(k_{\rho a} \rho\right) \hat{\mathbf{z}} \times \hat{\mathbf{p}}\right. \\
& \left.+\hat{\mathbf{p}} \cdot \hat{\boldsymbol{\phi}} \frac{2 k_{\rho a}}{j k_{z a}} J_{1}\left(k_{\rho a} \rho\right) \hat{\mathbf{z}}\right] e^{-j k_{z a} z} .
\end{aligned}
$$

The $\mathrm{D}$ contribution to the radiated electric field can also be calculated from $F_{\infty,+}^{D}$, according to (2) where, in the asymptotic limit, $\nabla \rightarrow j k \hat{\mathbf{u}}$, with $\hat{\mathbf{u}}$ denoting the local phase gradient (i.e., ray) direction, so that

$$
\mathbf{E}_{\infty}^{D}(\mathbf{r}) \sim \frac{2}{\pi k\left(\sin ^{2} \theta_{a}-\sin ^{2} \theta\right)} \frac{e^{-j k r}}{r} \hat{\mathbf{r}} \times \hat{\mathbf{p}}
$$

with $\hat{\mathbf{r}}=\sin \theta \hat{\boldsymbol{\rho}}+\cos \theta \hat{\mathbf{z}}$, and

$$
\mathbf{E}_{ \pm}^{D}(\mathbf{r})=\mp \sqrt{\frac{a}{2 \pi j k}} \frac{H_{0}^{(1)}\left(k_{\rho a} a\right)}{\sin \theta_{ \pm} \mp \sin \theta_{a}} \frac{e^{-j k r_{ \pm}}}{\sqrt{\rho r_{ \pm}}} \hat{\mathbf{r}}_{ \pm} \times \hat{\mathbf{p}}
$$

in which $\hat{\mathbf{r}}_{ \pm}=\sin \theta_{ \pm} \hat{\boldsymbol{\rho}}+\cos \theta_{ \pm} \hat{\mathbf{z}}$, and $\hat{\boldsymbol{\rho}}=\cos \phi \hat{\mathbf{x}}+\sin \phi \hat{\mathbf{y}}$. Fig. 2 schematically shows the different regions obtained as a result of the presented GTD analysis of transverse Bessel beam generation by an inward cylindrical traveling wave aperture distribution, polarized along a fixed unit vector. In particular, it is shown that the transverse Bessel beam (i.e., shaped as a zeroth order Bessel function) is radiated only in the yellow rhomboidal section conical region around the aperture axis.

In the next Section, the characterization of a planar slotted $\mathrm{CP}$ transverse Bessel beam launcher will be discussed.

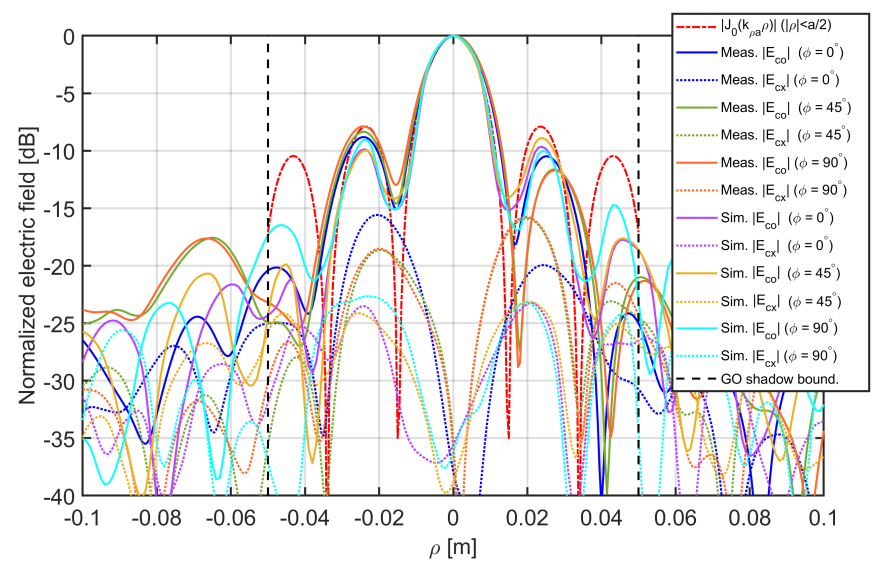

Fig. 4. Comparison of simulated and measured co-polar (RHCP, continuous lines) and cross-polar (LHCP, dotted lines) electric field component (with respect to the propagation $z$-axis) for different azimuthal cuts $\left(0^{\circ}, 45^{\circ}\right.$, and $90^{\circ}$ ), at $z=\mathrm{NDR} / 2=18.67 \mathrm{~cm}$, at the central frequency $30 \mathrm{GHz}$. The ideal Bessel function $J_{0}\left(k_{\rho a} \rho\right)$ is shown for comparison in the region $\theta<\theta_{a}$ (dashed-dotted line).

\section{ACCEPTED MANUSCRIPT}




\section{Prototype And Measurements}

In this Section, we present the fabrication of a Radial Line Slot Array (RLSA) Bessel beam launcher at the operating frequency $f=30 \mathrm{GHz}$, that radiates a $J_{0}\left(k_{\rho a} \rho\right) e^{-j k_{z a} z} \hat{\mathbf{z}} \times \hat{\mathbf{p}}$ profile in the transverse electric field component with respect to the propagation $z$-axis. According to the theory presented in Section II, by adopting the optimization scheme in [17], [22], [30] and the full-wave solver in [31], a RLSA has been designed which synthesize an equivalent magnetic current distribution as in (1), in which a right-hand circular polarization (RHCP) was chosen; i.e., $\hat{\mathbf{p}} \equiv \hat{\mathbf{p}}_{R H}=(\hat{\mathbf{x}}-j \hat{\mathbf{y}}) / \sqrt{2}$. A similar design could be repeated with linear polarization (LP) by arranging LP slot quadruplets [32], [33] in place of the $\mathrm{CP}$ slot pairs. To prove that a larger non-diffractive range can be achieved by the proposed transverse Bessel beam launcher with respect to the solution presented in [20], we assumed an axicon angle $\theta_{a}=15^{\circ}$, so that $k_{\rho a}=k \sin \theta_{a} \approx$ $162.62 \mathrm{rad} / \mathrm{m}$, and a circular radiating aperture of radius $a=10 \lambda=10 \mathrm{~cm}$, being $\lambda=1 \mathrm{~cm}$ the wavelength at the operating frequency. The non-diffractive range for such a launcher is then $\mathrm{NDR}=a \cot \theta_{a}=37.3 \lambda=37.3 \mathrm{~cm}$, that is greater than that in [20].

The optimized design of transverse RHCP Bessel beam launcher has been fabricated and measured in the bandwidth $29-31 \mathrm{GHz}$. The prototype is shown in Fig. 3. The radial PPW has been realized by using a ROGERS 5880 laminate, whose dielectric constant is $\epsilon_{r}=2.2$, loss tangent is $\tan \delta=9 \times 10^{-4}$, and thickness $h_{d}=2.54 \mathrm{~mm}$. Slot pairs have been etched on the PPW top plate by using laser ablation. The coaxial transition has been designed in the back side of transverse Bessel beam launcher, by using a standard coaxial connector (SRI Mod. 25-130-1000-90). As already done in [20], the impedance matching allover the considered bandwidth $29-31 \mathrm{GHz}\left(\left|S_{11}\right|<-10 \mathrm{~dB}\right)$ has been achieved by tuning both diameter $\left(D_{c}=2.48 \mathrm{~mm}\right)$ and height $\left(H_{c}=1.09 \mathrm{~mm}\right)$ of a cylindrical metallic cavity directly connected to the coaxial pin (inner diameter: $2 a_{c}=0.635 \mathrm{~mm}$, outer diameter: $2 b_{c}=1.46 \mathrm{~mm}$ ), as schematically shown in the inset of Fig. 3.

In Fig. 4, a comparison of simulated and measured co-polar (RHCP) and cross-polar (LHCP) electric field component (with respect to the propagation z-axis) is shown for different azimuthal cuts $\left(0^{\circ}, 45^{\circ}\right.$, and $\left.90^{\circ}\right)$, at $z=\mathrm{NDR} / 2=18.67 \mathrm{~cm}$, at the design frequency $30 \mathrm{GHz}$. The ideal Bessel function $J_{0}\left(k_{\rho a} \rho\right)$ is shown for comparison in the region $\theta<\theta_{a}$. The Bessel beam main lobe and also the first sidelobe are properly synthesized.

The field maps have been obtained by post-processing spherical near-field measurements. Namely, the field has been sampled over a measurement sphere surrounding the antenna under test (AUT) with radius $r=1.2 \mathrm{~m}$, and it has been used for calculating the spherical harmonics coefficients and the far-field pattern [34]. Then, from the far-field pattern, which corresponds to the field plane wave spectrum in the visible region, the radiative near-field has been retrieved on a set of planes parallel to the antenna via inverse Fourier transform [35]. Since the evanescent part of the spectrum is not

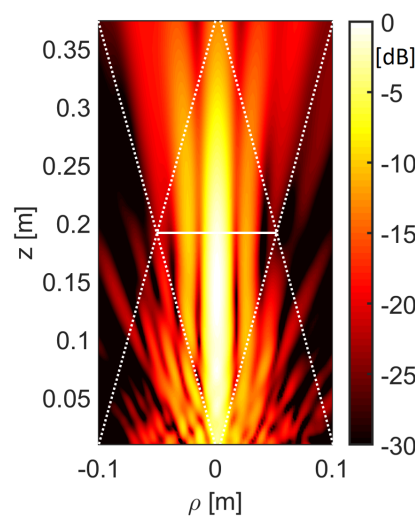

(a)

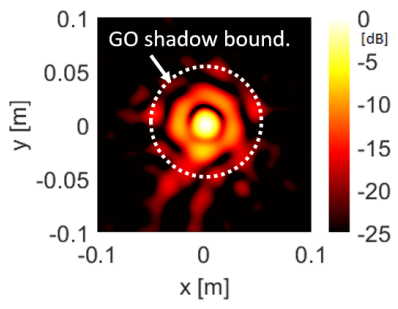

(c)

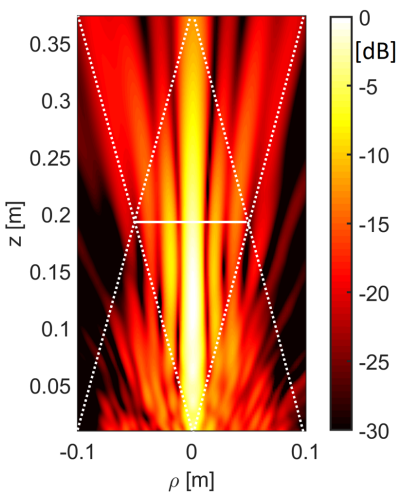

(e)

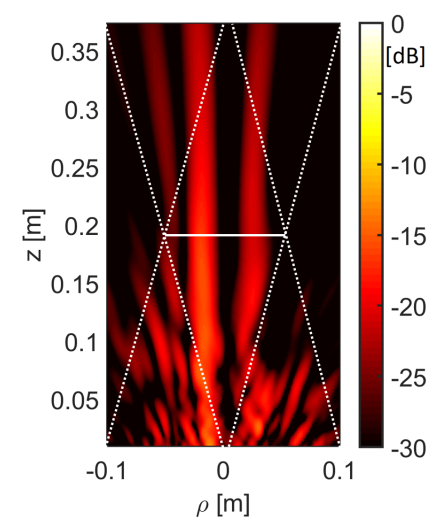

(b)

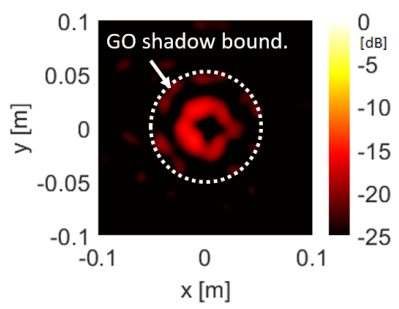

(d)

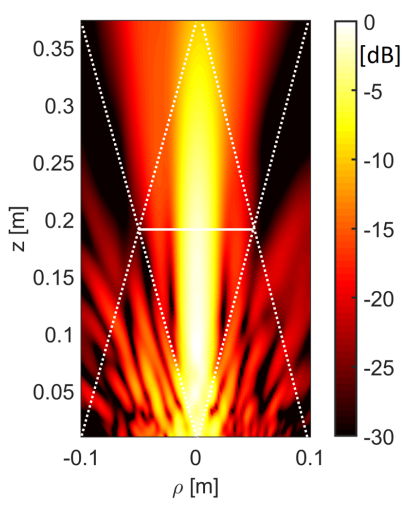

(f)
Fig. 5. Measured (a) co-polar (RHCP) and (b) cross-polar (LHCP) electric field transverse components (with respect to the propagation $z$-axis) in the $\rho-z$ plane $\left(\phi=0^{\circ}\right)$, at the design frequency $f=30 \mathrm{GHz}$. Measured (c) copolar (RHCP) and (d) cross-polar (LHCP) electric field transverse components (with respect to the propagation $z$-axis) in the transverse $x-y$ plane $(z=$ $\mathrm{NDR} / 2=18.67 \mathrm{~cm}$ ), at the design frequency $f=30 \mathrm{GHz}$. Measured copolar (RHCP) electric field transverse component in the $\rho-z$ plane $\left(\phi=0^{\circ}\right)$, at (e) $f=29 \mathrm{GHz}$ and (f) $f=31 \mathrm{GHz}$. The GO shadow boundaries, inside which the Bessel beam is radiated, are marked by a white dashed line.

known, the reactive field component cannot be recovered by applying such a technique, however such component becomes significant only very close to the antenna surface (usually at distance smaller than the operating wavelength).

In Fig. 5 measured (a)-(c) co-polar and (b)-(d) cross-polar electric field transverse components maps are shown at the design frequency in the longitudinal $\rho-z$ and in the transverse $x-y$ planes, at $z=\mathrm{NDR} / 2=18.67 \mathrm{~cm}$. Such a plot has been added to make a comparison with the theoretical results achieved in Section II, Fig. 2. Indeed, in the region

\section{ACCEPTED MANUSCRIPT}


$\theta<\theta_{a}$, the co-polar electric field transverse component recovers the shape of the target zeroth order Bessel shape, as theoretically predicted in Fig. 2 (yellow rhomboidal section conical region), whereas in the transverse $x-y$ plane the characteristic amplitude rings of Bessel beams arise inside the GO shadow boundary (white dotted circle). Finally, to prove that the Bessel beam is radiated also in a bandwidth around the design frequency, although the radiated beam constitutive parameters are in general frequency-dependent [26], in Fig. 5 measured co-polar (RHCP) electric field transverse component in the $\rho-z$ plane $\left(\phi=0^{\circ}\right.$ ) at (e) $f=29 \mathrm{GHz}$ and (f) $f=31 \mathrm{GHz}$ are shown.

\section{CONCLUSION}

We presented the analysis, design and fabrication of a limited-diffractive planar Bessel beam launcher, that exhibits a zeroth order Bessel profile in the transverse electric field component with respect to the $z$-propagation axis. The launcher has been designed by synthesizing a finite zeroth order, first kind Hankel aperture distribution, oriented along a fixed polarization unit vector $\hat{\mathbf{p}}$. The EM field radiated by such an aperture distribution has been derived by introducing an approximate model based on geometric theory of diffraction, hence it allowed to highlight relevant wave constituents involved in transverse Bessel beam generation (GO and D contributions), by taking into account also aperture finiteness. Moreover, the model has been validated by designing a CP planar transverse Bessel beam launcher, realized by means of a slotted radial waveguide, that is able to synthesize the above-mentioned aperture distribution. A prototype of RHCP transverse Bessel beam launcher has been then fabricated at $f=30 \mathrm{GHz}$. The transverse electric field component measurements in the bandwidth $29-31 \mathrm{GHz}$ have shown a satisfactory agreement with numerical full-wave simulations, and have finally proven the theoretical GTD-based model on transverse Bessel beam radiation.

\section{REFERENCES}

[1] J. Y. Lu and J. F. Greenleaf, "Ultrasonic nondiffracting transducer for medical imaging," IEEE Trans. ultrason. ferroelectr. freq. contr., vol. 37, n.5, 438-447, 1990.

[2] K. S. Lee and J. P. Rolland, "Bessel beam spectral-domain high-resolution optical coherence tomography with micro-optic axicon providing extended focusing range," Opt. Lett., vol. 33, n. 15, pp. 1696-1698, 2008.

[3] T. A. Planchon et al., "Rapid three-dimensional isotropic imaging of living cells using Bessel beam plane illumination," Nat. Methods, vol. 8, pp. 417-423, 2011.

[4] A. F. Valle and J. D. Seelig, "Two-photon Bessel beam tomography for fast volume imaging," Opt. Expr., vol. 27, n. 9, pp. 12147-12162, 2019.

[5] C. Liu, C. Jin, Y. Xiao, and H. Xie, "High-speed, multi-modal, label-free imaging of pathological slices with a Bessel beam," Biomed. Opt. Expr., vol. 11, n. 5, pp. 2694-2704, 2020.

[6] D. Mugnai, and P. Spalla, "Electromagnetic propagation of Bessel-like localized waves in the presence of absorbing media", Opt. Comm., vol. 282, pp. 4668-4671, 2009.

[7] A. Mazzinghi et al., "Large depth of focus pseudo-Bessel beam generation with a RLSA antenna," IEEE Trans. Antennas Propag., vol. 62, n. 8, pp. 3911-3919, 2014.

[8] J. Durnin, "Exact solutions for nondiffracting beams. I. The scalar theory," J. Opt. Soc. Am. A, vol. 4, pp. 651-654, 1987.

[9] J. Durnin, J. J. Miceli Jr., and J. H. Eberly, "Diffraction-free beams," Phys. Rev. Lett., vol. 58, p. 1499, 1987.

[10] M. R. Lapointe, "Review of non-diffracting Bessel beam experiments," Opt. Laser Technol., vol. 24, p. 315, 1992.
[11] J. Arlt and K. Dholakia, "Generation of high-order Bessel-beams by use of an axicon," Opt. Commun., vol. 177, p. 297, 2000.

[12] W. B. Williams and J. B. Pendry, "Generating Bessel beams by use of localized modes," J. Opt. Soc. Amer. A, vol. 22, pp. 992-97, 2005.

[13] Z. Li, K. B. Alici, H. Caglayan, and E. Ozbay, "Generation of an axially asymmetric Bessel-like beam from a metallic subwavelength aperture," Phys. Rev. Lett., vol. 102, p. 143901, 2009.

[14] O. Brzobohaty, T. Cizmàr, and P. Zemánek, "High quality quasi-Bessel beam generated by round-tip axicon," Opt. Expr., vol. 16, n. 17, pp. 12688-12700, 2008.

[15] M. Ettorre and A. Grbic, "Generation of propagating Bessel beams using leaky-wave modes," IEEE Trans. Antennas Propag., vol. 60, n. 8, pp. 3605-3613, 2012.

[16] M. Ettorre, M. Casaletti, G. Valerio, R. Sauleau, L. Le Coq, S. C. Pavone, and M. Albani, "On the near-field shaping and focusing capability of a radial line slot array," IEEE Trans. Antennas Propag., vol. 62, n. 4, pp. 1991-1999, 2014.

[17] M. Ettorre, S. C. Pavone, M. Casaletti, and M. Albani, "Experimental validation of Bessel beam generation using an inward Hankel aperture distribution," IEEE Trans. Antennas Propag., vol. 63, n. 6, pp. 25392544, 2014.

[18] M. Albani, S. C. Pavone, M. Casaletti, and M. Ettorre, "Generation of non-diffractive Bessel beams by inward cylindrical traveling wave aperture distribution," Opt. Expr., vol. 22, n. 15, pp. 18354-18364, 2014.

[19] S. C. Pavone, M. Ettorre, M. Casaletti, and M. Albani, "Transverse circular-polarized Bessel beam generation by inward cylindrical aperture distribution," Opt. Expr., vol. 24, n. 10, pp. 11103-11111, 2016.

[20] S. C. Pavone, M. Ettorre, and M. Albani, "Analysis and design of Bessel beam launchers: longitudinal polarization," IEEE Trans. Anten. Propag., vol. 22, n. 6, pp. 2311-2318, 2016.

[21] W. Fuscaldo, G. Valerio, A. Galli, et al., "Higher-order leaky-mode Bessel-beam launcher," IEEE Trans. Antennas Propag., vol. 64, n. 3, pp. 904-913, 2016.

[22] M. Albani, A. Mazzinghi, and A. Freni, "Automatic design of CP-RLSA antennas," IEEE Trans. Anten. Propag., vol. 60, pp. 5538-5547, 2012.

[23] M. Ettorre, S. C. Pavone, M. Casaletti, M. Albani, A. Mazzinghi, and A. Freni, "Near-field focusing by non-diffracting Bessel beams," in Aperture Antennas for Millimeter and Sub-Millimeter Wave Applications. Cham, Switzerland: Springer, 2018, pp. 243-288.

[24] W. Fuscaldo, D. Comite, A. Boesso, P. Baccarelli, P. Burghignoli, and A. Galli, "Focusing Leaky Waves: A Class of Electromagnetic Localized Waves with Complex Spectra," Phys. Rev. Appl., vol. 9, n. 5, p. 054005, 2018.

[25] S. C. Pavone, M. Ettorre, M. Casaletti, and M. Albani, "Analysis and design of CP Bessel beam launchers," 10th Europ. Conf. Antennas Propag. (EuCAP 2016), pp. 1-4, 2016.

[26] S. C. Pavone, A. Mazzinghi, A. Freni, and M. Albani, "Comparison between broadband Bessel beam launchers based on either Bessel or Hankel aperture distribution for millimeter wave short pulse generation," Opt. Expr., vol. 25, pp. 19548-19560, 2017.

[27] C. A. Balanis, Advanced Engineering Electromagnetics, John Wiley \& Sons, 2012.

[28] L. B. Felsen, and N. Marcuvitz, "Radiation and Scattering of Waves," Prentice-Hall, 1972.

[29] Z. Bouchal, and M. Olivik, "Non-diffractive Vector Bessel Beams," Journ. Modern Opt., vol. 42, n. 8, pp. 1555-1566, 1995.

[30] S. C. Pavone, M. Casaletti, and M. Albani, "Automatic Design of a CP Fan-Beam Linear Slotted Array in SIW Technology," IEEE Access, vol. 7, pp. 155977-155985, 2019.

[31] M. Casaletti, R. Sauleau, M. Ettorre, and S. Maci, "Efficient analysis of metallic and dielectric posts in parallel-plate waveguide structures," IEEE Trans. Microw. Theory Tech., vol. 60, n. 10, pp. 2979-2989, 2012.

[32] A. Mazzinghi, M. Albani, and A. Freni, "LP-RLSA Design for Low-Cost Transportable BASYLIS Radar [Antenna Applications Corner]," IEEE Antennas Propag. Magaz., vol. 55, n. 5, pp. 275-285, 2013.

[33] J. Takada, M. Ando, and N. Goto, "A Reflection Canceling Slot Set in a Linearly Polarized Radial Line Slot Antenna," IEEE Trans. Antennas Propag., vol. 40, n. 4, pp. 433-438, 1992.

[34] J. E. Hansen, "Spherical Near-field Antenna Measurements", IET, 1988.

[35] T. B. Hansen, A. D. Yaghjian, "Plane-Wave Theory of Time-Domain Fields: Near-Field Scanning Applications,” Wiley-IEEE Press, 1999.

\section{ACCEPTED MANUSCRIPT}

